\title{
Silicon Photonics: A Review
}

\author{
Ashish Dhiman \\ ${ }^{I}$ Department of Electronics and Communication, Delhi Technological University (Formerly Delhi College of \\ Engineering), Delhi, India)
}

\begin{abstract}
Previously, researchers were trying to replace the copper wires with the optical technology. They used photons instead of electrons to carry out the data within machines. The future of IC industry depends upon the speed and bandwidth requirements, but in metallic interconnections we have constraints on speed as well as bandwidth. With the advancement in computer technology we are now more dependent upon ultra fast data transfer rate between and within chips. Now, we can achieve high speeds with the use of optics, and if we integrate the optical components on a single silicon chip then it would allow the data to flow at a higher speed. This paper reviews the growth of silicon photonics compared to last few years and a promising future of photonics. Intel Corporation first announced its major breakthrough in 2004 based on silicon photonics (Moore's law). They developed a modulator based on silicon that operates at 50GHz. It was 50 times faster than the previous invention. In 2008 Intel declared that the data transfer rate can be increased further up to 1Tbps (bits per second).compatibility of silicon photonics with CMOS fabrication offers great advantages, such as low cost, high volume integration.
\end{abstract}

Keywords - Silicon, Modulators, Mach-Zehnder, SiGe, Detectors, Lasers, Avalanche Photodetector

\section{INTRODUCTION}

Silicon photonics is the new technology of producing optical devices and circuits using silicon as the core material for the integration of optical and electronic components on single chip with standard CMOS (complementary metal oxide semiconductor) fabrication process. Individual $\mathrm{Si}$ or $\mathrm{SiGe} / \mathrm{Si}$ components perform well at mid wave, long wave and very long -wave infrared $(\mathrm{THz})$ ranges. The intrinsic band gap of silicon (1.1 $\mathrm{eV})$ and its transparency at wavelengths $(1270 \mathrm{~nm}$ to $1625 \mathrm{~nm})$ typically favors for optical communication. It allows the integration of optical devices on silicon-on-insulator (SOI) wafers [1] which provides the route and manipulates the light. The present computers use copper wires to connect different components. These copper wires degrade the signal strength and have a limited maximum length. Intel's research achievement has replaced these connections with light weight optical fibres which can transfer more data over longer distances at higher speeds. Higher data rate can also be achieved through copper wires upto 10Gbps but with the increasing distance, speed starts decreasing.

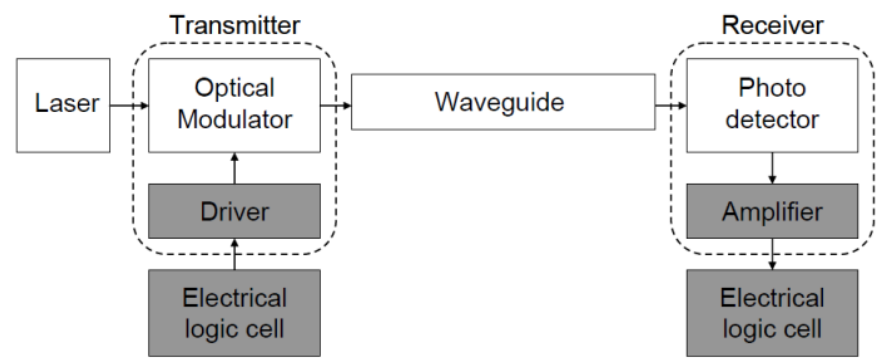

Fig(1): Basic block diagram of optical link [2]

Above fig (1) shows the basic diagram of optical link. Firstly, it requires an on-chip laser source which provides the pumping mechanism. Recently researchers focus on Hybrid Silicon Laser, in which the silicon is bonded to a different semiconductor (InP/GaAs) which acts as the lasing medium. To carry out the data in form of optical signals we require a Modulator. Modulation is carried out through free carrier plasma dispersion effect in photonics. Silicon waveguide provides the optical medium between different chip and same chip. At the receiver end metal semiconductor junctions based photodetectors integrated into silicon waveguides for detection of light and converting back the data into electronic signals. In order to get the higher data rate over long distances, one possible solution is to use optical interconnections with silicon as an optical medium. In electrical medium signal information carried by the electrons but in optical medium information carried by the photons. Comparison to the electrons, photons has no charge and zero rest mass therefore photons can travel at higher rate i.e. speed of light and better stability. Therefore it is desirable to replace electrical circuits with optical circuits 


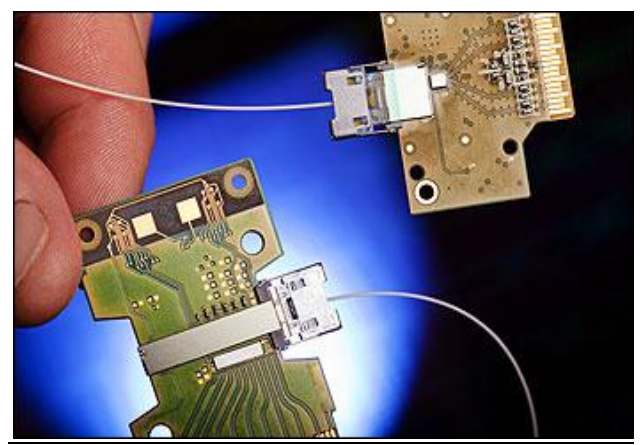

Fig(2): 50 Gbps silicon photonics transmit module [3]

Above fig(2) explains the 50Gbps optical link module generated by the Intel group (2007) [3]. Silicon chip presence at the centre of the board (lower side) provides laser light which travels through optical fibre, where a second silicon chip (upper side) detects the data on the laser and converts it back into an electrical signal. Optoelectronic systems integration on silicon on substrate (SOI) realizable with complementary metal-oxide semiconductor (CMOS) technology, allows low cost and large scale manufacturing for Si photonic devices. Further work going on the development of integration methods of low loss waveguides, high quality resonators, high speed modulators and optically pumped lasers on silicon chip for low cost and power consumption effective photonic circuits. This highly combination of Photonic technology fulfils the demands of numerous fields such as communication, computing, imaging and sensing. The most recent advancement involves encoding of high-speed data on an optical beam. This paper describes the photonic structures that are liable for Si based optic-electro communication link.

\section{HIGH-SPEED SILICON MODULATION}

An Optical Modulator is a device which is used to modulate a beam of light with respect to an information signal. Various factors decide the performance of the modulators: (1) modulation depth (2) modulation speed (3) bandwidth. In ideal cases, we prefer high modulation speed, large bandwidth and low power consumption.

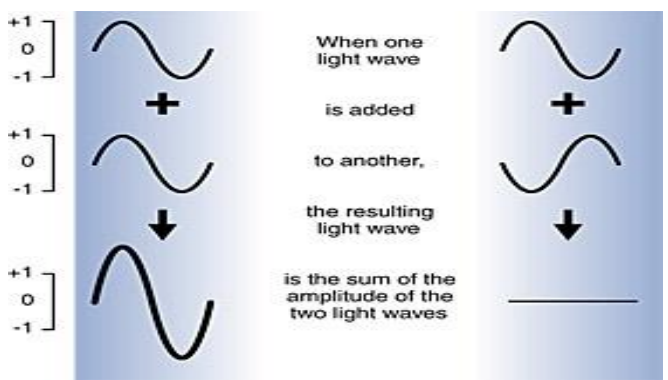

Fig(3): Modulation process of wave [4]

Fig (3) illustrates the modulation process where two wavelengths are combined. If two sine waves are perfectly matched (sync) then they are added together, resulting sine wave has twice the amplitude of the individual waves. In contrast, when two waves are completely out of sync, then resulting wave has no amplitude because two light waves cancel each other out [4]. Silicon is not considered to exhibit good electro-optic effect that could enable the modulation process but it favors the fabrication of various micro-optical devices. Silicon has transparency to infrared communication wavelength and has high refractive index which allows the miniaturization of photonic devices. In silicon integrated circuits modulation is come through by free carrier plasma dispersion effect.

Various constraints such as bandwidth, narrow waveguide, modulation efficiency, low power consumption is kept in mind while integrating optical modulators on silicon substrate. Keeping in that mind recently, researchers reduced the waveguide core size from the micrometer range, producing silicon nanowires with a height and width of about 500nm and less [5]. 

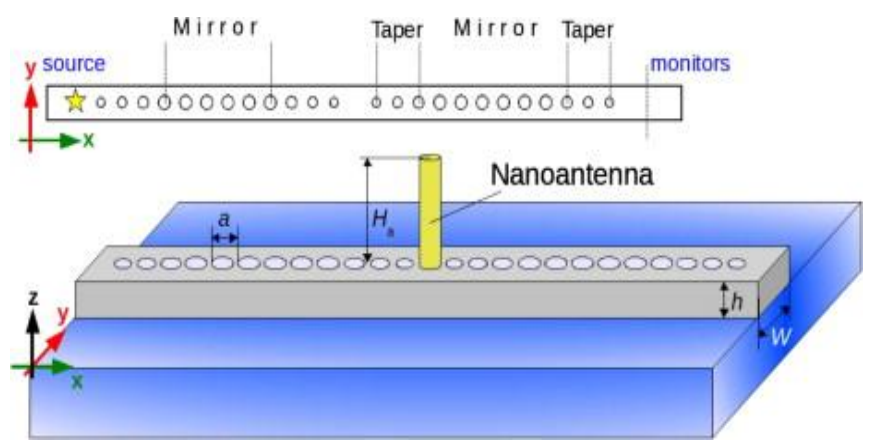

Fig(4): Illustrates the silicon-on-insulator nano-beam cavity with a plasmonic nano-antenna.

The cavity is formed by two tapered mirrors consisting of air holes in silicon rectangular nanowire (gray region, width $\mathrm{W}=500 \mathrm{~nm}$ and height $\mathrm{h}=260 \mathrm{~nm}$ ) with a silica substrate (blue region). The distance between the holes of the mirrors is $\mathrm{a}=375 \mathrm{~nm}$ and the radius of the holes is $\mathrm{r}=0.28 \mathrm{a}$. The radii of the holes in the tapers are $0.24 \mathrm{a}$, $0.22 \mathrm{a}$ and $0.19 \mathrm{a}$, respectively. The diameter and the height of the gold nano-antenna are $\mathrm{D}=135 \mathrm{~nm}$ and $\mathrm{Ha}=500 \mathrm{~nm}$, respectively [6]. Previously, we can achieve the speed up to $\sim 10 \mathrm{Gbps}$ through silicon modulators but with the increasing demand of fast data rate we have to use the efficient and fast modulators. For high speed (>10Gbps), optical modulators based on electro-optic materials such as III-V semiconductors or LiNbO3 [7] are used in recent years. With these modulators, one can achieve modulation upto 50Gbps. It is not easy to achieve the high modulation because of crystalline structure of silicon, as it does not exhibit the linear electro-optic Pockels[8] effect and has a very weak Franz-Keldysh effect, Kerr effect [9,10] at optical communication wavelengths of $1.3 \mu \mathrm{m}$ and $1.6 \mu \mathrm{m}$. Silicon also has high thermal coefficient ( 2E-4)[11] which is not suitable for high speed operation of modulators. Recently, it has been shown that strained silicon $(\mathrm{Ge} / \mathrm{SiGe})$ structures have strong electro-optic effect due to quantum-confined Stark effect[12] making it desirable for optical modulation. Combining, modulators with recently developed hybrid silicon lasers, one could create a single chip that can transmit data at $1 \mathrm{Tbps}$ data rate. For fast optical modulation, free carrier plasma dispersion effect is considered, which is related with the concentration of free carriers in a semi-conductor, which changes both real and imaginary part of the refractive index. Change in the refractive index due to plasma dispersion effect can be used for modulation in two ways (1) Phase shifter /modulator (2) Resonant structures. Phase shifter /phase modulation configuration depends upon MachZehnder interferometer (MZI) which is used to determine the relative phase shift from the variations of refractive index in one or both arm of wave-guide. MZI configuration describes the two optical modulators structures based on carrier depletion and carrier accumulation mode.

\section{Metal-oxide semiconductor (MOS ) Capacitor-Based Silicon Modulator}

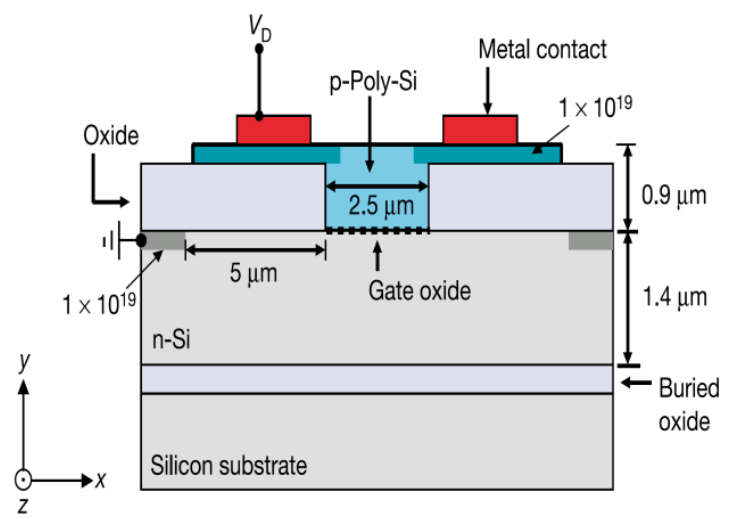

Fig(5): Cross-section view of the MOS capacitor-based on silicon optical modulator.

In case of this modulator, modulation is achieved by driving the MOS capacitor in the accumulation mode of operation. Positive voltage $V_{D}$ applied on the gate, which forms the depletion and inversion layer across the waveguide region. Due to this positive voltage large amount of silicon charge carriers accumulate in the depletion region which changes the refractive index. Change in refractive index further changes the phase shift and attenuation in one or both arms of interferometer. 

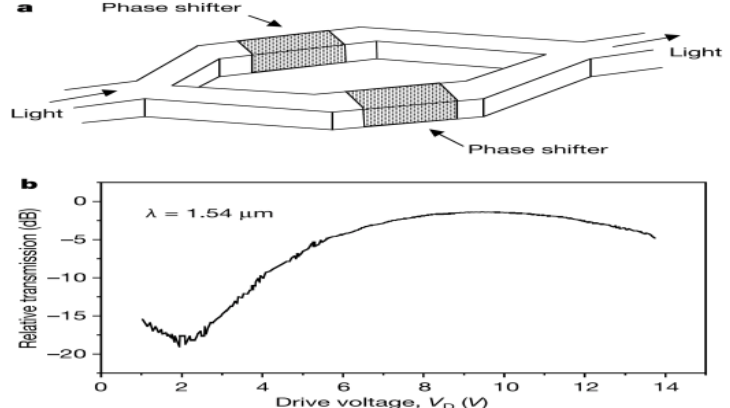

Fig(6): a) Phase shifter in both arms of MZI, b) Drive voltage change with relative transmission

When light enters into MZI it will split up into two beams in both arms of the interferometer. Phase shifter changes the relative phase shift between the two beams and modulation is achieved on the output of interferometer. Changes in the phase shift and attenuation in the arms of interferometer generated from the plasma dispersion effect. MOS capacitor also improves the modulation efficiency. Speed of charge carriers in MOS capacitor is independent upon minority carrier's lifetime but depends upon the device resistance and capacitance. The optical loss of the phase shifter in high-speed section is $10 \mathrm{~dB} / \mathrm{cm}$ and $5.2 \mathrm{~dB} / \mathrm{cm}$ for the lowspeed sections. MZI modulator gives a total insertion loss of $19 \mathrm{~dB}$ consisting of $9 \mathrm{~dB}$ coupling loss and $10 \mathrm{~dB}$ on-chip loss. Out of $10 \mathrm{~dB}$ on-chip loss, $3.5 \mathrm{~dB}$ is due to the high-speed (RF) sections, $2.5 \mathrm{~dB}$ due to the lowspeed (bias) sections[13]. Phase efficiency can be further be improved by reducing the waveguide dimensions and reducing the gate dielectric width. First breakthrough in MOS capacitor shown by Intel in 2004, with modulation bandwidth exceeding $1 \mathrm{GHz}[14]$.

\section{P-N Or P-I-N BASED SILICON MODULATOR}

Modulation is achieved in these modulators by operating the p-n diode[15] in reverse bias and in the carrier depletion mode. It is also based on MZI configuration and improvement of the MOS capacitor based modulators. Because of the reverse bias it requires low input power and independent of minority carrier life time i.e. no speed problem. But its main drawback is its low modulation depth which occurs due to overlap between the active electrical region and optical region.

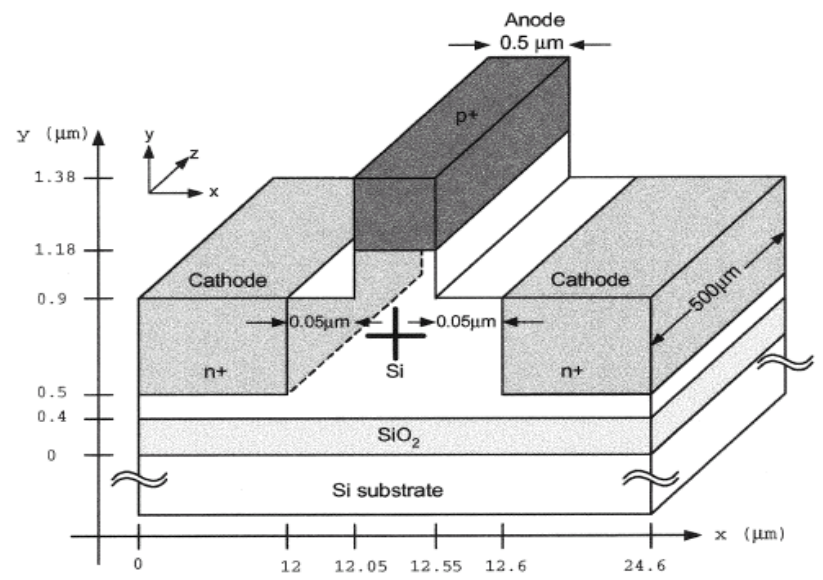

Fig(7): p-i-n Si Optical Modulator

In this modulator, the dielectric layer between two doped regions replaced with the p-n junction. This structure arrangement reduces the modulator capacitance hence improved the bandwidth. The concentrations of $n$ type are higher than the $\mathrm{p}$ type results carrier depletion mainly occurs in $\mathrm{p}$ type. It leads to better phase modulation efficiency because the hole density change results in a larger refractive index change as compared to the electron density change as indicated by[16]. Phase shift provided by the reverse biased p-n junction embedded in MZI arms shown in fig(8) 


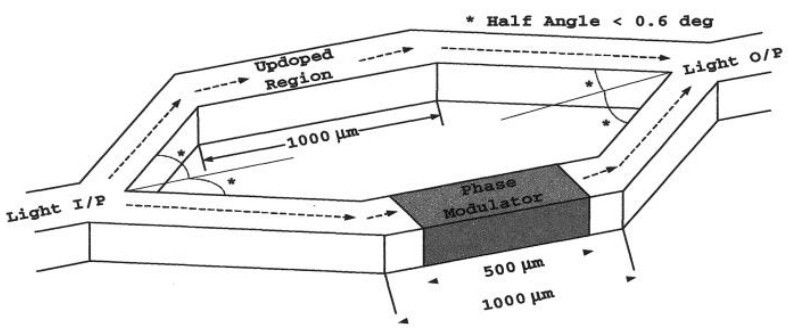

Fig(8): Phase modulator embedded in MZI arms.

The first carrier depletion-based silicon modulator built by Gardes et al. in 2005[17], bandwidth of 50GHz. After few years, Basak et al. in Intel came through with modulator (p-n diode based) with data rate of $40 \mathrm{Gbps}$ and bandwidth of $30 \mathrm{GHz}[18,19]$. Although carrier depletion modulators have higher speed operation but it requires improvement in the modulation depth.

\section{Energy Harvesting}

Sasan Fathpour and Bahram Jalali gave us another idea regarding optical modulators i.e. energy harvesting in silicon modulators. It is based on the consumption of pump energy that is being lost in two photon absorption (TPA) into some useful electrical power. For TPA to happen strong optical intensity light source is required. Modulation is carried out through electrical losses caused by TPA[20].The input optical power and wire cross-section are kept in such that optical intensity is high enough to induce the TPA. TPA induced free carriers give us V-I characteristics similar to the solar cell. These devices give us electrical power whenever biased in the fourth quadrant.

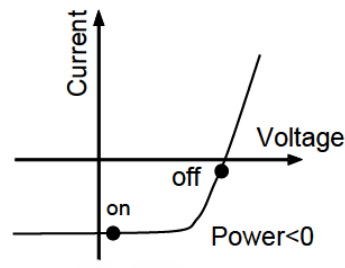

Fig(9): V-I characteristics in the fourth quadrant with negative power dissipation [20]

Above fig(9) shows that whenever device in fourth quadrant electrical power generated and device performs as a modulator otherwise energy is dissipates in the form of heat. Energy harvesting is significant for VLSI industry where high number of devices integrate on single chip resulting large dissipation of heat.

\section{Electro-absorption modulators}

Electro absorption modulators are a semiconductor device that modulates the intensity of laser beam via electric voltage. Principle of operation based on the Franz-Keldysh effect. Compared to plasma dispersionbased modulators, absorption type modulators have high operating speed, low power dissipation and low operating voltage. Through these modulators a modulation bandwidth of $10 \mathrm{GHz}$ can be achieved. Recently, it has been shown that strained silicon $(\mathrm{GeSi})[21]$ or $\mathrm{Ge} / \mathrm{GeSi}[22]$ multiple quantum wells also exhibits linear electro-optic refractive index modulation.

\section{Hybrid Silicon Modulator}

Efficient modulators should have large bandwidth, high speed operation and good modulation efficiency. Modulators based on MZI have bandwidth close to $100 \mathrm{~nm}$ while electro-absorption modulators are less than 30nm. Therefore for high speed operation, hybrid silicon modulators are developed, where the III-V material epitaxial layer grow on silicon-on-insulator (SOI) wafer by wafer bonding techniques[23]. LiNbO3 is the most widely used material as electro-optic modulators. It has high electro-optic coefficient and mature fabrication technology making it suitable for modulation applications. Previously, LiNbO3 modulators have modulation speed of 20Gbps but now ultrafast LiNbO3 of 60Gbps have been developed by titanium (Ti) diffusing [7]. 


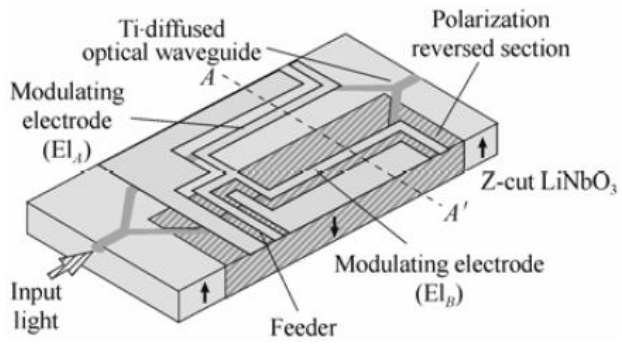

Fig(10): LiNbO3 modulator with Ti diffusing[24]

Input signal is provided by the feeder which is further divided into two signals on the modulating electrodes E1A and E1B with same phases and amplitude. Phase shift provided by the arms of MZ interferometer in opposite direction. These Modulators were fabricated by the polarization reversal technique[24,25,26]. Modulators based on MachZehnder (MZ) or Electro-Absorption (EA) provides data rate higher than 10Gbps whereas MachZehnder structures on Lithium-Niobate (LiNbO3) give us data rate up to 60Gbps. To achieve higher data rate various new modulations format such as OPSK (Quadrature Phase Shift keying), DQPSK (Differential Quaternary Phase-Shift Keying)[27] are under progress. Recently, these formats based LiNbO3 modulator give us 80Gbps data rate. Currently, researcher's works on the multi-level modulation format based on MQAM (Quadrature-Amplitude Modulation) applied on single carrier to achieve the data rate of 100Gbps or beyond. Another approach supporting the higher data rate is the division of transmitted signal into sub carriers. These sub carriers are aligned orthogonally with the same symbol rate. Recently, Intel has also demonstrated 50Gbps optical link and their future work of achieving 1Tbps based on the sub carrier's modulation[3].

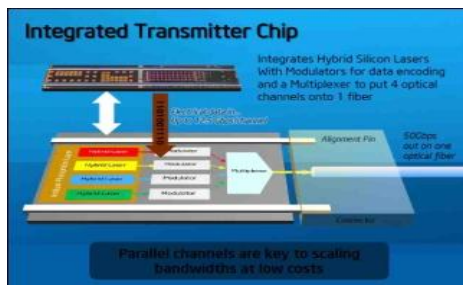

(a)

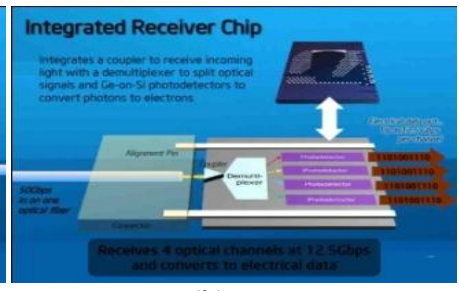

(b)

Fig(11): Showing (a) Transmitter (b) Receiver with wavelength division multiplexing

Transmitter chip includes four hybrid silicon lasers (InP), generates four different colors of continuous-wave laser light or wavelengths. Wavelength division multiplexing (WDM) increases the number of channels in the fiber which increases the bandwidth. From the four hybrid silicon lasers, light beams travel into an optical modulator that encodes data on to them at $12.5 \mathrm{Gbps}$. These four beams further combined to a single optical fibre for a total data rate of $50 \mathrm{Gbps}$. At the receiver link, chip separates the four optical beams and directs them into photo detectors, which convert them back into electrical signals. Based on this model, research has been going on to achieve data rate of $100 \mathrm{Gbps}$ or further.

\section{Photodetectors}

Photodetectors is the semiconductor devices which convert the optical signals into electrical signals. Photodetectors should have high sensitivity in operating wavelength, high response speed, low noise, high reliability and low biasing across the detector. Photoconductor is the simplest and basic detector consists of single semiconductor slab across which electric field is applied. But it has high dark current noise and high response speed. Instead of this reverse biased pn junction has low dark current noise. Therefore photo detectors consist of $\mathrm{p}-\mathrm{n}$ junction, works under a reverse bias voltage. Reverse bias voltage across the pn junction increases the reverse current and width of the depletion region. Thickness of the depletion region further increases the quantum efficiency but the response time increases. Photo detector performance mainly depends upon three factors (1) Responsivity (2) Dark current (3) Bandwidth.

Dark current is the small electric current shown by the detector under no input light signals. It is also referred as reverse leakage current in detectors. Responsitivity determines the receiving efficiency of the detectors and calculated as

$$
P=\eta \frac{e}{h \nu}
$$


Where $\mathrm{h} v$ is the photon energy, $\eta$ is the quantum efficiency, and e is the elementary charge. Efficiency can be increased by adding an intrinsic area in pn junction. When device operates in reverse biased then width of depletion region becomes equal to the intrinsic region. Therefore it increases the light absorption area and increases the quantum efficiency. Quantum efficiency can also be increased by using Anti-reflection coating on the surface. Bandwidth of the photo detectors depends upon junction capacitance and the generation of carriers across the junction. Bandwidth related to junction capacitance $(\mathrm{Cj})$ given as

$$
f_{B W}=1 /\left(2 * \pi * R_{L O A D} * C_{j}\right)
$$

With the increase in depletion width due to reverse, capacitance of the depletion region increases therefore bandwidth (response speed) increases. In most cases, detectors can have larger responsivity, but it will also increase both the dark current and the capacitance and hence reduces the sensitivity and the bandwidth, respectively[28]. Semiconductor materials, such as $\mathrm{Si}$, Ge, and III-V material, like GaAs, InP, have been used for the manufacturing of hetrojunction photodetectors. Figure(12) compares the absorption coefficients of different semiconductors including Silicon. From this fig(12) it can be seen that $\mathrm{Si}$ and GaAs have very low absorption coefficient while Ge and some other III-V materials have considerable absorption coefficient

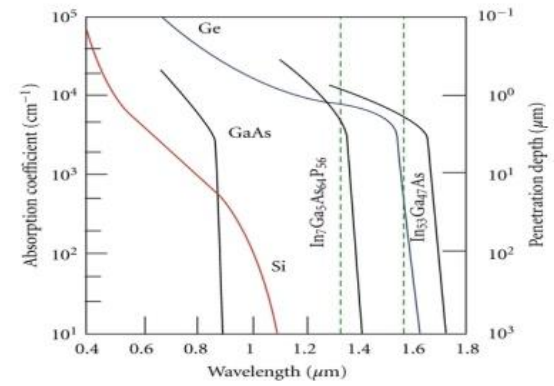

Fig(12): Absorption coefficient as a function of their wavelength. The green dashes mark typical wavelengths for telecommunications windows of 1310 and $1550 \mathrm{~nm}$ [29]

\section{Silicon Detectors}

From the fig(12) it is clear that $\mathrm{Si}$ exhibit transparency in the $1300-1550 \mathrm{~nm}$ operating wavelength range because of its low absorption coefficient it is not a suitable material for fibre-optic communication application. But it performs well under $1000 \mathrm{~nm}$, where band-to-band absorptions happen. To solve this problem, we can approach to three structures; Schottky structure, III-V heterogeneous detectors, or Ge detectors built on silicon substrates.

\section{Schottky Detectors}

Schottky detectors are also known as metal semiconductor diode, consists of a thin metal layer $(\sim 100$ $\AA$ A) on a lightly doped p-type $\mathrm{Si}$, forming a Schottky barrier at the material interface. Schottky barrier results the depletion layer between the metal and semiconductor junction also known as the absorption region. The barrier is usually $0.2-0.6 \mathrm{eV}$ for $\mathrm{p}-\mathrm{Si}$, while the value is a bit smaller for $\mathrm{n}-\mathrm{Si}$ with the same contact metal. Main advantages of Schottky detectors that it is majority carrier device, not dependent on minority carrier's lifetime therefore no delays problems. It has also high response speed $(150 \mathrm{GHz})$ and compatible with standard CMOS technology. But its drawback is the low quantum efficiency. Quantum efficiency can be improved by merging the dielectric waveguide using transparent conducting electrodes and employing surface Plasmon polaritons (SPP) [30,31]. Fig(11) shows below symmetric and asymmetric surface Plasmon Schottky contact detector. Scales et al. demonstrated symmetric structure with responsivity of $0.1 \mathrm{~A} / \mathrm{W}$ and dark current of $21 \mathrm{nA}$ [32].

In the symmetric structure, fabrication process is complicated as it involves the realization of thin metal films buried in semiconductor in order to simply the fabrication process Berini`s group proposed the asymmetric Surface Plasmon polaritions photodetectors[33,34]. 

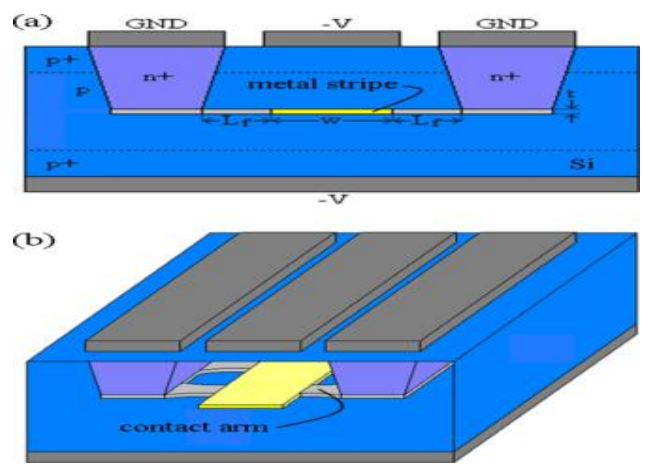

Fig(13): Scheme diagram of examples of (a) symmetric and (b) asymmetric surface Plasmon Schottky contact detector.

\section{III-V Heterogeneous Detector}

III-V semiconductors have direct band gap and compared to silicon III-V-based detectors have wider absorption bandwidth and relative low dark currents. But the lattice structures of III-V materials are different from the Si therefore these semiconductor materials cannot be directly grown on silicon. One solution to this problem is the heterogeneous integration of III-V layers on silicon substrate using advanced wafer bonding technique. In earlier times III-V detectors (GaAs,) are not compatible with CMOS technology approach. But in recent times some of them, like InGaAs/InP built on the SOI substrate using heterogeneous integration approach. This integration process achieved by the DVS-BCB adhesive die-to-wafer bonding process [35].In fig (12) metal-semiconductor-metal photo detector (MSM) using an InGaAs absorption layer were realized. Coupling is formed between the SOI waveguide and photo detector by the vertical directional coupling between the SOI waveguide and the lossy III-V waveguide mode. The dark current of the device was 3.0nA at a bias voltage of $5 \mathrm{~V}$, while the responsivity of detector is $1.0 \mathrm{~A} / \mathrm{W}$ at $1.55 \mu \mathrm{m}$ and a bias voltage of $5 \mathrm{~V}[36]$.

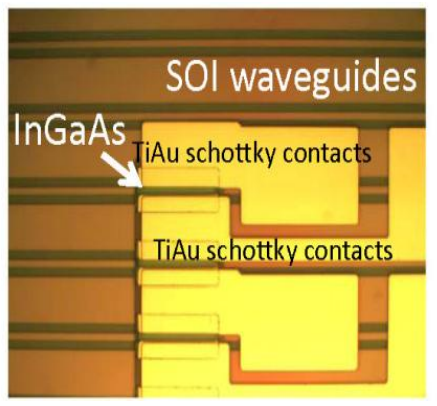

(a)

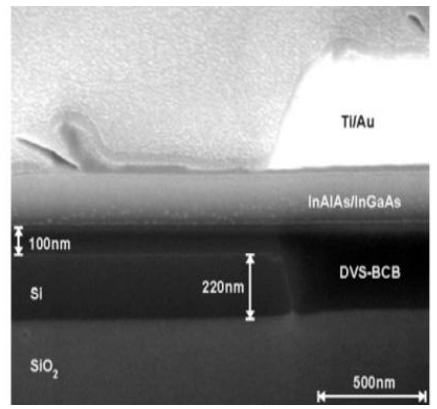

(b)

Fig(14): (a) Top view of the fabricated III-V metal-semiconductor-metal photodetectors integrated on an SOI waveguide platform (b) cross-section of the device.

\section{Germanium Detector built on Silicon}

Ge has indirect bandgap structure similar to Si but its band gap energy is less $(0.7 \mathrm{ev}$ versus $1.1 \mathrm{ev}$ for $\mathrm{Si}$ ) compared to $\mathrm{Si}$. Therefore it has higher absorption coefficient up to wavelength of 1550nm [29]. Today, most of the researchers work on Ge-on-Si photodetectors because of its high responsivity behavior in near IR wavelength, its optoelectronic properties and compatibility with CMOS fabrication. Ge/GeSi can be deposited by chemical vapour deposition process (CVD). In these photodetectors main difficulty comes in growing o epitaxial films of Ge on $\mathrm{Si}$. Due to the high lattice constant of Ge $4 \%$ greater than the silicon, there is always mismatched in their structures. This will cause defects while growing Ge layers on $\mathrm{Si}$ such as dislocations in their densities arrangement and high power loss. These defects greatly affect the performance of the photodetectors. To overcome with this problem we have two different approaches (1) selectively growing of Ge on Si (2) two step method of growing Ge. In the selective approach we generally use Si3N4 as the dielectric mask through which Ge layers grow on the silicon. Deposition of layers of Ge is done through chemical vapour deposition (CVD) through the exposed areas develop over the dielectric mask[1,9]. In two step method Ge layers grow epitaxially on $\mathrm{Si}$ under controlled temperature. Initially, Ge layer of $30 \mathrm{~nm}$ grown on $\mathrm{Si}$ at a temperature of $320-360^{\circ} \mathrm{C}$. In the second step continuous growth of Ge desired over the $\mathrm{Si}$ at a higher temperature greater than $600^{\circ} \mathrm{C}$. Later on Loh et al. [37] demonstrated modified two step approach, which includes growing of ultrathin $(2 \sim 30 \mathrm{~nm})$ Low Temperature SiGe buffer layer prior to the deposition of Low Temperature Ge layer and High Temperature Ge layer. 
There are two structures of Ge-on-Si p-i-n photo detectors (1) normal-incidence type (2) waveguide type. Waveguide based photo detectors is better than normal-incidence photo detectors on the basis of quantum efficiency and bandwidth. Waveguide detectors have smaller area than the incidence type, about ten times. There has been great progress going on Ge on Si photo detectors. Vivien et al. demonstrated the Ge detector with bandwidth of $42 \mathrm{GHz}$ and responsivity at $1 \mathrm{~A} / \mathrm{W}$ operating at $1550 \mathrm{~nm}$ wavelength[38]. Feng et al. also showed the zero bias Ge detector with $17.5 \mathrm{GHz}$ bandwidth[39]. CMOS integration process lowers down the bias voltage across the detectors, reduce the dark current, power absorption and operating voltage of chip. Recently Luxtera[40] and MIT[41] demonstrated Ge detectors of $130 \mathrm{~nm}$ and $180 \mathrm{~nm}$ respectively integrated with CMOS technology Currently, a zero bias Ge detector with $17.5 \mathrm{GHz}$ has been demonstrated by. In 2010, Kang group reported Ge detector with high sensitivity at $4 \mathrm{~dB}$ and speed at $10 \mathrm{~Gb} / \mathrm{s}$.

\section{Avalanche Photodetector}

Avalanche photodiode detectors (APD) have better sensitivity than the p-i-n type detectors and exhibit larger gain bandwidth product. Ge-on-Si APD gives us better performances than the III-V detectors. In a avalanche based photodetectors number of charge carriers increases by 10-100 factor due to multiplication of charge carriers. Therefore it increases the sensitivity, bandwidth and helpul in long distance transmission. Progress work going on the development of low cost Ge-on-Si APD with data rate of 40Gbps. Recently the Intel-UCSB team has demonstrated a $340 \mathrm{GHz}$ gain-bandwidth $\mathrm{Ge}-\mathrm{Si}$ avalanche photodiode using CVD growth of $\mathrm{Ge}$ and $\mathrm{Si}$ layers at $850^{\circ} \mathrm{C}[42]$.

\section{LIGHT EMITTERS AND LASER}

Si has an indirect bandgap structure which implies that radiative recombination requires emission or absorption of phonon. The involvement of phonon makes it very inefficient light emitter. Several progresses have been made in silicon based light emitters and laser sources. Initially researchers focus on the quantum dot lasers and fusing of impurities into silicon such as neodymium but these attempts are not useful. Later on researchers work on some other alternatives. In 2002[43] ,idea was proposed of using Stimulated Raman Scattering (SRS) in silicon waveguides.Two years later, Jalali's group demonstrated the first Si laser based on Raman Effect at UCLA. But it has free carrier absorption (FCA) and mean carrier lifetime problems which make it impossible to achieve the gain in silicon waveguides. FCA occurs when a material absorbs a photon and a carrier is excited from a filled state to an unoccupied state (in the same band). FCA is removed by integrate the waveguide structure into the intrinsic region of a PIN diode, which is reverse biased so that the carriers are attracted away from the waveguide. In 2005[44], Intel Corp. reported the first continuous-wave (CW) Si Raman laser based on reversed biased p-i-n Si waveguide structure. Si properties like high optical damage threshold, high thermal conductivity and large reflective index contributes the silicon as an excellent element for Raman crystal. Si Raman laser operate well in $1550 \mathrm{~nm}$ near-IR band but it has one problem i.e. the loss induced by twophoton absorption (TPA) and Free-carrier absorption (FCA). TPA is a nonlinear loss mechanism, it occurs when two photons combine their energies to boost an electron in the valence band to the conduction band. Silicon Raman lasers are considered to be the ideal light sources because of its unmatched wavelength purity and the possibility of extending the lasing wavelength into the mid-infrared region. Researchers also found that the action of silicon nanocrystals on erbium ions give us a new base for silicon based optical amplifiers and laser sources. The main advantages of erbium-doped silicon is (1) easy to fabricate(2) CMOS compatible(3) wavelength suitable for optical communication(4) optical activity demonstrated. For improving the emitter efficiency multi layered structures of Er doped Si nanocrystals preferred. Efficiency is also dependent upon the doping concentration and device geometry. Main problem with erbium doped silicon is that it forms the Auger process because erbium is electrically active and its high pumping mechanisms.

There is another light source in which III-V-based materials (GaAs and InP) are grown epitaxially on silicon substrate. Compared to $\mathrm{Si}, \mathrm{GaAs}$ and $\mathrm{InP}$ have direct bandgap structure which favors the laser mechanism. But the major problem is large lattice mismatch between III-V-based materials (GaAS and InP) and Silicon which results dislocation of densities of 10E $8-10 \mathrm{E} 10 \mathrm{~cm}-2$ [45] respectively. Another disadvantage is III-V materials epitaxial growth methods are not compatible with CMOS processing results large power loss. Various approaches have been made to remove the dislocations such as direct wafer bonding, strained super lattices [46], divinyltetramethyl-disiloxanebenzocyclobutene (DVS-BCB) adhesive bonding[7]. 


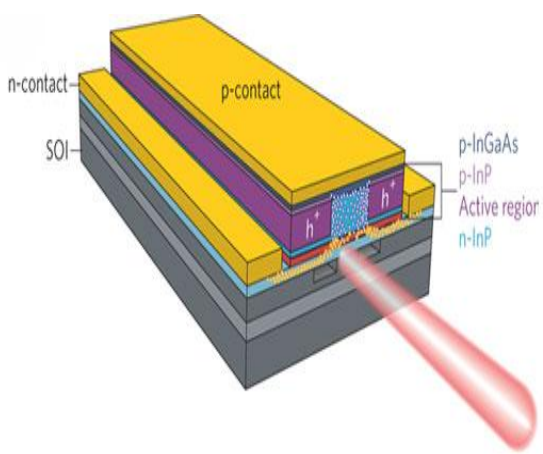

Fig(15): Injected carriers in iii-v epitaxial layers on top of a Si waveguide.

To overcome with the problem of III-V-based materials growth on silicon substrate, researchers now work on the optimized growth of III-V materials under low temperatures. One of the examples is InGaAs nanopillar (NP) laser compatible with CMOS processes. Another useful approach is $\mathrm{Ge}$-on-Si (or SiGe-on-Si) epitaxial growth. $\mathrm{Ge}$ and $\mathrm{Si}$ both has mismatch in their lattice constant and thermal coefficients. Germanium has an direct band but it has the chances of radiative recombination because of its lower energy gap (0.8eV). High thermal coefficients of Ge also favor the radiative recombination because of reduction in tensile strain of is $0.2-0.25 \%$ [47]. These are the basis of direct bandgap electroluminescence and optically pumped operation of Ge-on-Si lasers. MIT demonstrated the first Ge on Si continuous wave laser working at room-temperature, fabricated by epitaxial growth of $1.6 \mu \mathrm{m} * 0.5 \mu \mathrm{m}$ Ge waveguides on $\mathrm{Si}[48]$.
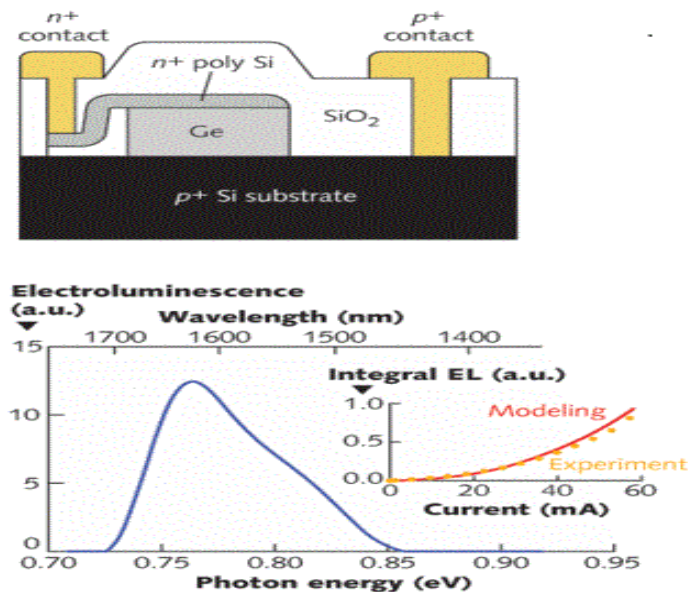

Fig(16): Ge epitaxial growth on Si and the electroluminescence behavior.

Instead of growing single material on substrate researchers developed the new formation where combinations of several materials grown on silicon substrate simultaneously. Hybrid silicon laser is a semiconductor device where III-V group materials fabricated on silicon substrate. III-V group materials have direct band gap structure compared to Si therefore in hybrid approach we use the light emitting properties of III-V materials with mature fabrication technique. In 2006, first electrical driven Hybrid Silicon Laser was developed with collaboration between the University of California, Santa Barbara, and Intel Corporation[49]. These lasers do not require any optical input and it can directly fabricate on to silicon chip. In the hybrid laser unpatterned III-V wafer is bonded to a patterned silicon wafer with optical waveguides. Silicon waveguides are patterned before laser fabrication so no alignment is needed between the unpatterned III-V wafer and the patterned silicon wafer.

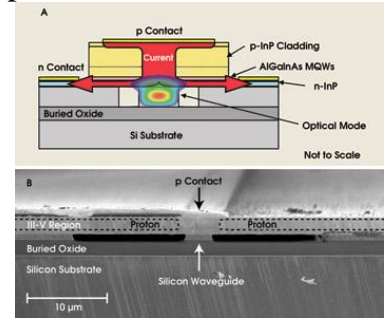

Fig(17): Hybrid Silicon Laser (a) and the scan view (b)

When light or electrical signals drives the hybrid laser then light emits from active layer of AlGaInAs quantum 
wells in the III-V material couples into the silicon waveguide. In Hybrid Silicon Laser, III-V material used for optical gain/modulation and silicon used for optical wave-guiding medium. Gain can be varied by adjusting both the height and the width of the silicon waveguide and it is dependent upon the overlapping between the hybridwaveguide mode and the quantum-well region. Using this approach, a variety of discrete active devices such as distributed feedback (DFB), distributed Bragg reflector (DBR), ring fabry-perot and AWG lasers have been fabricated. Currently, Hybrid III-V on Si technology is the most advanced photonic integrated circuits on Si. Researchers now working on the new structures of hybrid laser e.g. Ring or disk geometries for hybrid laser. First electrically pumped hybrid Si micro-ring laser was demonstrated by Liang et al.[50].

(a)
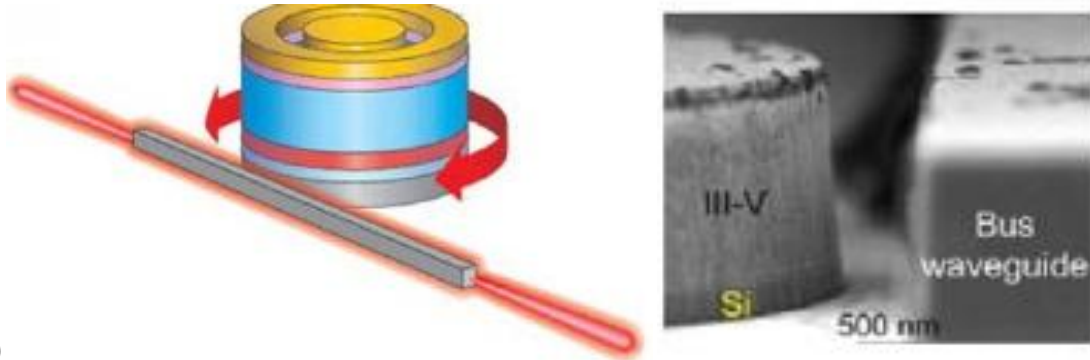

(b)

Fig(18): (a) Hybrid micro-ring laser with a Si bus waveguide. (b)SEM image of resonator sidewall and bus waveguide

\section{APPLICATIONS AND FUTURE ASPECTS}

Silicon photonics, extremely powerful technology in the recent trades, fulfills the demands for higher speed, efficiency, and low power consumption at lower costs. In silicon technology, various opto-electronics devices integrate on a single substrate connected with each other using narrow waveguides. Such circuits could be used to establish high speed transmission, increased bandwidth, reduced power consumption and decreasing latency problems. It can potentially increase the bandwidth capacity by providing micro-scale, ultra low power devices. Optical data transmission increases the data rate and eliminates the problem of electromagnetic interference. With these data rates one could imagine the videoconferencing with a high resolution that the actors or family members appear to be in the room with you. Optical link can transfer data over longer distances and faster than today's copper technology; up to 50 GB of data per second. Recently, Intel Corporation launched their optical link connection operating 100Gbps[51]. Fujitsu Laboratories recently developed four wavelength integrated silicon laser for optical transrecievers. Work is in the progress for the new optic-interconnection of external devices to PCs. Intel introduced Light Peak, 2009 which replaces the USB and communicates the data at up to $10 \mathrm{Gbps}$. Advancement in the field of silicon nanophotonic technologies leads new ideas in future computing systems and their architectures.

\section{DRAWBACKS}

(1) Silicon's main drawback is its poor optical emission efficiency due to its indirect band-gap. Silicon has inefficient electroluminescence; therefore it suffers from light sources. For these reasons, R\&D focuses on the development of practical silicon light sources. Lasers are preferred as a light source but it requires high pumping mechanism. Significant progress has been made in the field of hybrid silicon laser which allow low cost and provides efficient laser light. (2) Optical connections require precise alignment therefore it requires improved alignment technologies.(3) Heat dissipated by the laser source on chip.(4) Band-gap of silicon is

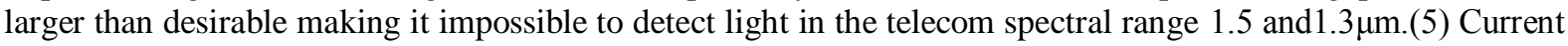
fabrication processes are localized for making pure electronics devices. Integrating photonic components on the same chip requires additional fabrication steps that may interrupt the standard process and affect the performance of the electronic components. (6) Integration of components increasing day by day on chip therefore power dissipation of components also increases across the chip.

\section{REFERENCES}

[1] R. A. Soref.: 'The past, present, and future of silicon photonics', IEEE J. Sel. Top. Quantum Electron, 12, (6), pp. 1678- 1687, 2006.

[2] Ali Okey, Thesis, SI-GE Photodetection Technologies for integrated optoelectronics, partialfulfilment of doctraldiss, Stanford University, 2007.

[3] Http://www.intel.com/pressroom/archive/releases/2010/20100727comp_sm.htm

[4] Dr. Mario Paniccia, Victor Krutul, Sean Koehl, Intel Unveils Silicon Photonics Breakthrough: High-Speed Silicon Modulation, Technology@Intel Magazine, 2004.

[5] Frederic Gardes, GoranMashanovich, GrahamReed,"Evolution of optical modulation in silicon-on-insulator devices", SPIE Newsroom. DOI: 10.1117/2.1200712.0985, 2007.

[6] Ivan S. Maksymov,"Optical switching and logic gates with hybrid plasmonic-photonic crystal nanobeam cavities "Physics Letters A $375,2011918-921$. 
[7] K. Noguchi, "Ultra-high-speed LiNbO3 modulators," Journal of Optical and Fiber Communications Reports, vol. 4,no. 1, pp. 113,2007 .

[8] R.S Jacobsen,K.N Anderson ,P.I.Borel,ETAl.,"Stranded Silicon as a New Electrooptic material, "Nature, Vol.441, No.7090.PP.199202.2006 .

[9] R. A. Soref and J. P. Lorenzo, "All-silicon active and passive guided-wave components for $\lambda=1.3$ and $1.6 \mu \mathrm{m}$," IEEE Journal of Quantum Electronics, vol. 22, no. 6, pp. 873-879, 1986.

[10] R. A. Soref and B. R. Bennett, "Electro optical effects in silicon," IEEE Journal of Quantum Electronics, vol. 23, No. 1, pp. 123$129,1987$.

[11] G. T. Reed and A. P. Knights, Silicon Photonics: An Introduction, John Wiley \& Sons, Chichester, UK, 2004.

[12] Y.-H. Kuo, Y. K. Lee, Y. Ge, et al., "Strong quantum-confined Stark effect in germanium quantum-well structures on silicon," Nature,vol. 437, no. 7063, pp. 1334-1336, 2005.

[13] L. Liao, A. Liu, R. Jones, et al., "Phase modulation efficiency and transmission loss of silicon optical phase shifters," IEEE Journal of Quantum Electronics, vol. 41, no. 2, pp. 250-257, 2005.

[14] Ansheng Liu1, Richard Jones1, Ling Liao1, Dean Samara-Rubio1, Doron Rubin2, Oded Cohen2, Remus Nicolaescul \& Mario Paniccia1,"A high-speed silicon optical modulator based on a metal-oxide-semiconductor capacitor", Nature427, 615-618,2004.

[15] C. K. Tang, G. T. Reed, "Highly efficient optical phase modulator in SOI waveguides," Electron. Lett. 31, 451-452,1995.

[16] Http://www.intel.com/products/processor/core2XE/qc_prod_brief.pdf.

[17] F. Y. Gardes, G. T. Reed, N. G. Emerson, and C. E. Png, "A sub-micron depletion-type photonic modulator in silicon On insulator, "Optics Express, vol. 13, no. 22, pp. 8845-8854, 2005.

[18] L. Liao, A. Liu, D. Rubin et al., "40 Gbps silicon optical modulator for high-speed applications," Electronics Letters, vol. 43, no. 22,pp. 1196-1197, 2007.

[19] A. Liu, L. Liao, D. Rubin et al., "Recent development in a high-speed silicon optical modulator based on reverse-Biased pn diode in a silicon waveguide," Semiconductor Science and Technology, vol. 23, no. 6, Article ID 064001, 2008.

[20] S. Fathpour and B. Jalali, "Energy harvesting in silicon optical modulators," Optics Express, vol. 14, no. 22, pp. 10795-10799, 2006.

[21] J. Liu, M. Beals, A. Pomerene, S. Bernardis, R. Sun, J. Cheng, L. Kimerling, and J. Michel, "Waveguide-integrated, ultralowenergy GeSi electroabsorption modulators," Nat. Photonics 2(7), 433-437, 2008.

[22] Y. H. Kuo, Y. K. Lee, Y. Ge, S. Ren, J. E. Roth, T. I. Kamins, D. A. Miller, and J. S. Harris, "Strong quantum-confined Stark effect in germanium quantum-well structures on silicon," Nature 437(7063), 1334-1336 , 2005.

[23] D. Liang, G. Roelkens, R. Baets, and J. Bowers, "Hybrid integrated platforms for silicon photonics," Materials 3(3), 1782-1802, 2010.

[24] Ichikawa J, Oikawa S, Yamamoto F, et al. Zero chirp broadband Z-cut LiNbO3 optical modulator using polarization reversal and branch electrode. Proceedings of 29th Optical Fiber Communication Conference (OFC'04): Vol 1, Feb 23-27, 2004, Los Angeles, CA, USA.Piscataway, NJ, USA: IEEE, 2004.

[25] Courjal N, Porte H, Martinez A, et al. "LiNbO3. Mach-Zehnder modulator with chirp adjusted by ferroelectric domain inversion" IEEE Photonics Technology Letters, 14(11): 1509-1511, 2002.

[26] Murata H, Kaneda K, Yamamoto S." Low-power and low-chirp guided-wave electrooptic intensity modulator by use of domaininverted structure" Proceedings of Conference on Lasers and Electro-Optics (CLEO’03), Baltimore, MD, USA. Piscataway, NJ, USA: IEEE, 2003: 1008-1010, 2003.

[27] Lu G W, Miyazaki T, Ichikawa J, et al. High-speed DQPSK transmitter using a monolithically integrated quad Mach-Zehnder IQ modulator driven at quarter bit-rate. Proceedings of the 34th European Conference on Optical Communication (ECOC'08), Sep 21-25, 2008, Brussels, Belgien. Piscataway, NJ, USA: IEEE, 2008.

[28] Zhou Fang, Ce Zhou Zhao," Recent Progress in Silicon Photonics: A Review”, ISRN Optics Volume 2012 , Article ID 428690,2012 .

[29] B. Jalali, M. Paniccia, and G. Reed, "Silicon photonics," IEEE Microwave Magazine, vol. 7, no. 3, pp. 58-68, 2006.

[30] Reather, H., 1988, Surface Plasmons on Smooth and Rough Surfaces and on Gratings. Springer, Berlin, 1988.

[31] Ebbesen, W., Lezec, H. J., Ghaemi, H. F., Thio, T., Wolff, P. A., Extraordinary optical transmission through sub-wavelength hole arrays, Nature, 391, 667-669,1997.

[32] C. Scales, I. Breukelaar, and P. Berini, "Surface-plasmonSchottky contact detector based on a symmetric metal stripe in silicon, “Optics Letters, vol. 35, no. 4, pp. 529-531, 2010.

[33] Akbari A., and Berini, P., Schottky contact sur-face-plasmon detector integrated with an asymmetric metal stripe waveguide, Appl. Phys. Lett., 95(2), 021104,2009.

[34] A. Liu, R. Jones, L. Liao, D. Samara-Rubio, D. Rubin, O. Cohen, R. Nicolaescu, and M. Paniccia, "A high-speed silicon optical modulator based on a metal-oxide-semiconductor capacitor," Nature 427(6975), 615-618, 2004.

[36] J. Brouckaert et al., "Compact InAlAs/InGaAs metal semiconductor-metal photodetectors coupled with SOI waveguides, submitted for publication in Photonics Technology Letters".

[37] T. H. Loh, H. S. Nguyen, C. H. Tung et al., "Ultrathin low temperature SiGe buffer for the growth of high quality Ge epi layer on $\mathrm{Si}(100)$ by ultrahigh vacuum chemical vapor deposition,” Applied Physics Letters, vol. 90, no. 9, Article ID 092108, 2007.

[38] L. Vivien, J. Osmond, J. M. Fédéli et al., " $42 \mathrm{GHz}$ p.i.n germanium photodetector integrated in silicon-on-insulator waveguide,"Optics Express, vol. 17, no. 8, pp. 6252-6257, 2009.

[39] D. Feng, S. Liao, P. Dong et al., "High-speed Ge photodetector monolithically integrated with large cross-section silicon-oninsulator waveguide," Applied Physics Letters, vol. 95, no. 26, Article ID 261105, 2009.

[40] G. Gunn, "CMOS Photonics for high-speed interconnects," Journal Microelectronics, vol. 26, pp. 58-66, 2006.

[41] M. Beals, J. Michel, J. F. Liu et al., "Process flow innovations for photonic device integration in CMOS," in Silicon Photonics III, vol.6898 of Proceedings of SPIE, San Jose, Calif, USA, January 2008.

[42] Kang, Y.M., Liu, H.D., and Morse, M., et al.: "Monolithic germanium/ silicon avalanche photodiodes with $340 \mathrm{GHz}$ gainbandwidth product", Nature Photonics, 3, (1), pp. 59-63, 2009.

[43] Jalali, B., Raghunathan, V., and Dimitropoulos, D., et al.: "Raman based silicon photonics", IEEE J. Sel. Top. Quantum Electron, 12, (3), pp. 412-421, 2006.

[44] Rong, H.S., Jones, R., and Liu, A.S., et al.: “A continuous-wave Raman silicon laser”, Nature, 433, (7027) ,pp. 725-728, 2005.

[45] Kawanami, H. ,"Hetero-epitaxial technologies of III-V on Si”. Sol. Energy. Mat. Sol. C. 66, 479-486, 2001.

[46] Masafumi, Y., Mitsuru, S. \& Yoshio, I. "Misfit stress dependence of dislocation density reduction in GaAs films on Si substrates grown by strained-layer super lattices". Appl. Phys. Lett. 54, 2568-2570, 1989. 
[47] Liu, J. et al. "Tensile-strained, n-type Ge as a gain medium for monolithic laser integration on Si" Opt. Express 15, 11272-11277, 2007.

[48] Liu, J., Sun, X., Camacho-Aguilera, R., Kimerling, L. C. \& Michel, J. Ge-on-Si laser operating at room temperature. Opt. Lett. 35, 679-681, 2010.

[49] Fang, A.W., Park, H., and Cohen, O., et al.: "Electrically pumped hybrid AlGaInAs-silicon evanescent laser", Opt. Express, 14, (20),pp. 9203-9210, 2006

[50] D. Liang, M. Fiorentino, T. Okumura, H.H. Chang, D.T. Spencer, Y.H. Kuoetal.”Electrically-pumped compact Hybrid silicon microring lasers for optical interconnects" Optics Express, pp. 20355-20364, 2009.

[51] D. McGrath, "Intel is developing optical chip-to-chip interconnects," Electronic Engineering Times, vol. 1556, p. $39,2009$. 